\title{
O.A. YESHCHENKO
}

Faculty of Physics, Taras Shevchenko National University of Kyiv

(4, Prosp. Academician Glushkov, Kyiv 03127,Ukraine; e-mail: yes@univ.kiev.ua)

The temperature dependences of the energy and the width of a surface plasmon resonance are studied for copper nanoparticles 17-59 $\mathrm{nm}$ in size in the silica host matrix in the temperature interval 293-460 K. An increase of the temperature leads to the red shift and the broadening of the surface plasmon resonance in Cu nanoparticles. The obtained dependences are analyzed within the framework of a theoretical model considering the thermal expansion of a nanoparticle, the electron-phonon scattering in a nanoparticle, and the temperature dependence of the dielectric permittivity of the host matrix. The thermal expansion is shown to be the main mechanism responsible for the temperature-induced red shift of the surface plasmon resonance in copper nanoparticles. The thermal volume expansion coefficient for Cu nanoparticles is found to be size-independent in the studied size range. Meanwhile, the increase of the electron-phonon scattering rate with the temperature is shown to be the dominant mechanism of the surface plasmon resonance broadening in copper nanoparticles.

Ke ywords: surface plasmon resonance, copper nanoparticles, temperature-induced effects

\section{Introduction}

Noble metal nanoparticles exhibit unique optical properties such as the resonant absorption and the scattering of light, which are not found in bulk counterparts $[1,2]$. Collective coherent oscillations of the free electrons in the conduction band, also known as a Surface Plasmon (SP) resonance, are responsible for the strong absorption and the scattering of light by particles [1]. The energy and the width of an SP resonance depend on the size, morphology, spatial orientation, and optical constants of particles and the embedding medium $[1,2]$. The excitation of an SP resonance in a metal nanoparticle leads to an enhanced local electric field close to the surface of the particle [1-4]. Therefore, noble metal nanoparticles have attracted a lot of attention recently due to a wide range of potential applications in the surface enhanced Raman scattering (SERS) [5-7], surface enhanced fluorescence [8-10], biochemical imaging [11-13], can-

(C) O.A. YESHCHENKO, 2013

ISSN 2071-0194. Ukr. J. Phys. 2013. Vol. 58, No. 3 cer treatment [11,14-16], and subwavelength optical waveguides [17-21], to name just a few.

An influence of the temperature on the SP resonance in metal nanoparticles is crucial for the pure and applied science of nanoparticles $[1,22]$. The temperature dependence of the SP resonance is important because of the recent applications of noble metal nanoparticles to the thermally assisted magnetic recording [23], thermal cancer treatment [16,2426 ] catalysis and nanostructure growth [27], and computer chips [28]. However, the SP temperature dependence was not studied in detail to date, because a broad temperature interval requires a usage of materials with high thermal stability. Colloids, thin films, and glasses, which are major media in optical materials with metal nanoparticles, possess no enough thermal stability (800-900 K at maximum for glasses). The more advanced material which is highly transparent in the optical range is silica, and the facile sol-gel technique allows one to produce metal nanoparticles of the different chemical nature and the size range within the matrix that is stable in air up to $1600 \mathrm{~K}$. 
In the present work, we use the silica sol-gel glasses admitting the usage of a wider temperature interval without the sample destruction. For these samples, the full reversibility in the variation of optical properties occurred.

The underlying physics of the temperature dependence of optical properties of metal nanoparticles is a precondition for the development of successful and reliable applications and devices. The temperature effects for the SP resonance absorption band in metal nanoparticles were studied, e.g., by Kreibig [1, 29], Doremus [30,31], and the origin of temperature effects upon the SP resonance was analyzed by Mulvaney [32]. Recently, the influence of the temperature on the $\mathrm{SP}$ resonance in $\mathrm{Au}$-based plasmonic nanostructures was reported for low temperatures $80-400 \mathrm{~K}$ in Ref. [33], where the appreciable red shift and the SP resonance broadening with increasing temperature were observed. The temperature-induced broadening of the SP extinction band leaded to an appreciable decrease of the light exctinction at the SP resonance frequency and, respectively, to an increase of the extinction on the wings of the SP extinction band. The interplay of temperature effects and a material quality in nanoscale plasmonic waveguiding structures was discussed in Ref. [34]. So, most of the effects related to the temperature dependence of the SP resonance were observed for low temperatures $[1,33]$. Therefore, there is a lack of data on the influence of the temperature on an SP resonance in metal nanostructures for temperatures higher than room one. The temperature dependence of the SP resonance energy and bandwidth for gold colloidal nanoparticles was studied by Link and El-Sayed [35] for temperatures higher than room one. Gold colloidal nanoparticles within the size range from $9 \mathrm{~nm}$ to $99 \mathrm{~nm}$ were studied at elevated temperatures up to $365 \mathrm{~K}$. No significant influence of the temperature on the SP resonance energy and bandwidth was found. In our recent work [36], we studied the temperature dependence of the SP resonance in silver nanoparticles at high temperatures in the silica matrix similar to that in the present work. We observed the noticeable red shift and the broadening of the SP resonance in Ag nanoparticles with increasing temperature, which is in full accordance with effects reported for plasmonic Au-nanostructures at low temperatures.

In this paper, we present experimental results on the temperature dependence of the SP resonance en- ergy and width in copper nanoparticles 17-59 $\mathrm{nm}$ in size embedded in the silica matrix in the temperature interval 293-460 K. We have observed that an increase of the temperature of a sample leads to the red shift and the broadening of the SP resonance, which is similar to our results [36] obtained for $\mathrm{Ag}$ nanoparticles in silica at high temperatures and to results of Bouillard et al. [33] obtained for Au nanostructures at low temperatures. We analyze the observed temperature dependence of the SP resonance within the framework of a theoretical model considering such phenomena as the thermal volume expansion of a nanoparticle, electron-phonon scattering in a nanoparticle, and temperature dependence of the dielectric permittivity of the host matrix. We show that the main mechanism of the red shift of the SP resonance with increasing temperature is the thermal volume expansion of a nanoparticle, while the electronphonon scattering in a nanoparticle is the dominant mechanism of SP resonance broadening. The paper is organized as follows. After the introduction, we give a short description of the procedure of synthesis of copper nanoparticles and a structural and optical characterization of nanoparticles. In Section 3, we describe the experimental observations of the red shift and the broadening of the SP resonance, as the temperature increases. In Section 4, we outline a theoretical model of the temperature dependence of the SP resonance energy and width. In Section 5 , we give the comparative analysis of experimental data and results of theoretical calculations and discuss the significance of different mechanisms leading to the temperatureinduced red shift and the broadening of the SP resonance in copper nanoparticles. In Conclusions, we summarize the main obtained results.

\section{Synthesis of Copper Nanoparticles and Their Optical and Structural Characterization}

Composite $\mathrm{Cu} / \mathrm{SiO}_{2}$ samples with copper nanoparticles were produced, by using the modified sol-gel technique based on hydrolysis of tetraethoxysilane (TEOS) with Cu-doping followed by a chemical transformation of the precursors of dopants under annealing in air or controlled gaseous medium. A precursor sol was prepared by the mixing of TEOS, water, and ethyl alcohol with the acid catalyst such as $\mathrm{HNO}_{3}$ or $\mathrm{HCl}$. The silica powder with a particle size of about 
$5-15 \mathrm{~nm}$ (the specific surface area is $380 \pm 30 \mathrm{~m}^{2} / \mathrm{g}$ ) was added to the sol followed by ultrasonication in order to prevent a large volume contraction during drying. The next gelation step resulted in the formation of gels from sols. Porous materials (xerogels) were obtained, after the gels were dried at room temperature. The porosity of $\mathrm{SiO}_{2}$ matrices was controlled by annealing the samples in air at $600{ }^{\circ} \mathrm{C}$ during $1 \mathrm{~h}$. Doping by copper was performed by immersion of xerogels into a $\mathrm{Cu}\left(\mathrm{NO}_{3}\right)_{2}$ alcohol solution during $24 \mathrm{~h}$. Then the impregnated samples have been dried in air at $40{ }^{\circ} \mathrm{C}$ during $24 \mathrm{~h}$. The further processing of the $\mathrm{Cu}$-doped xerogels was done by two pathways. (1) The initial annealing in air with gradual increase of the temperature from $20{ }^{\circ} \mathrm{C}$ to $1200{ }^{\circ} \mathrm{C}$ (annealing time at $1200{ }^{\circ} \mathrm{C}: 5 \mathrm{~min}$ ), then the annealing in the atmosphere of molecular hydrogen at a temperature of $800{ }^{\circ} \mathrm{C}$ during $1 \mathrm{~h}$. (2) An annealing in $\mathrm{H}_{2}$ with gradual increase of the temperature from $20{ }^{\circ} \mathrm{C}$ to $1200{ }^{\circ} \mathrm{C}$ (annealing time at $1200{ }^{\circ} \mathrm{C}: 5 \mathrm{~min}$ ). The annealing resulted in the decomposition of $\mathrm{Cu}\left(\mathrm{NO}_{3}\right)_{2}$ followed by the nucleation and the aggregation of $\mathrm{Cu}$ clusters resulting in the formation of $\mathrm{Cu}$ nanoparticles of various sizes. Glass samples fabricated were polished up to a thickness of about $1 \mathrm{~mm}$ for optical measurements. The sets of samples were prepared. The samples obtained at the successive annealing in air and hydrogen are light-pink colored (samples AH); ones obtained at annealing in hydrogen are red colored (samples $\mathrm{H})$.

The copper nanoparticles formed were characterized with transmission electron microscopy (TEM) to determine their mean size and morphology. TEM characterization was performed with the use a JEOL JEM-2000EX electron microscope. Figure 1 shows the typical TEM micrographs of some samples. It can be seen that TEM indicates a large separation between nanoparticles. Therefore, the electrodynamical coupling cannot affect their optical spectra, and we use one-particle models for simulations below. The micrographs prove the creation of spherical $\mathrm{Cu}$ nanoparticles of various sizes in the matrix in dependence on the annealing conditions. Nanoparticles with mean sizes of $17 \mathrm{~nm}$ and $35 \mathrm{~nm}$ are formed in the samples annealed successively in air and then in molecular hydrogen (samples $\mathrm{AH}$ ). $\mathrm{Cu}$ nanoparticles with mean sizes of $48 \mathrm{~nm}$ and $59 \mathrm{~nm}$ are formed in the samples annealed in $\mathrm{H}_{2}$ (samples $\mathrm{H}$ ). TEM shows that $\mathrm{Cu}$ nanoparticles in the studied samples are charac-
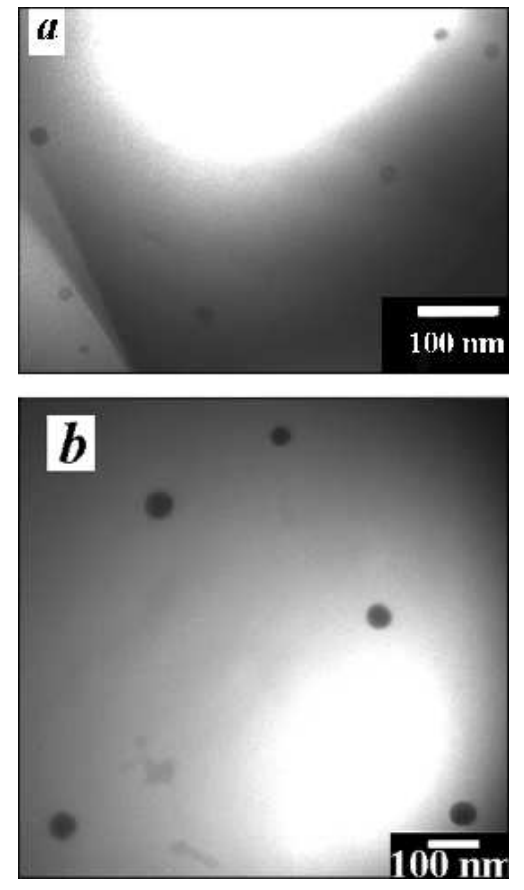

Fig. 1. Typical TEM micrographs of $\mathrm{Cu}$ nanoparticles in the silica matrix. $a-\mathrm{AH} 2$ sample annealed successively in air and hydrogen $(\langle d\rangle=17 \mathrm{~nm}), b-\mathrm{H} 1$ sample annealed in hydrogen $(\langle d\rangle=48 \mathrm{~nm})$

terized by the Gaussian size distribution with clearfeatured maximum. The dispersion of the size distribution is quite low in all the samples being in range of 11-17\% depending on the sample. Since the distribution dispersion is small, it is reasonable to conclude that the effect of the particle size distribution would not affect the shape and the width of the SP absorption band of copper nanoparticles.

A tungsten-halogen incandescent lamp was used as a light source in the measurements of absorption spectra. A single grating spectrometer MDR-3 was used for the registration of spectra. The samples were placed into an open furnace during the spectral measurements. Each spectrum was measured at its own stabilized temperature.

\section{Temperature Dependence of Surface Plasmon Resonance in $\mathrm{Cu}$ Nanoparticles: Experiment}

We measured the absorption spectra of copper nanoparticles in the silica host matrix. Samples containing $\mathrm{Cu}$ nanoparticles with mean sizes of 17,35 , 


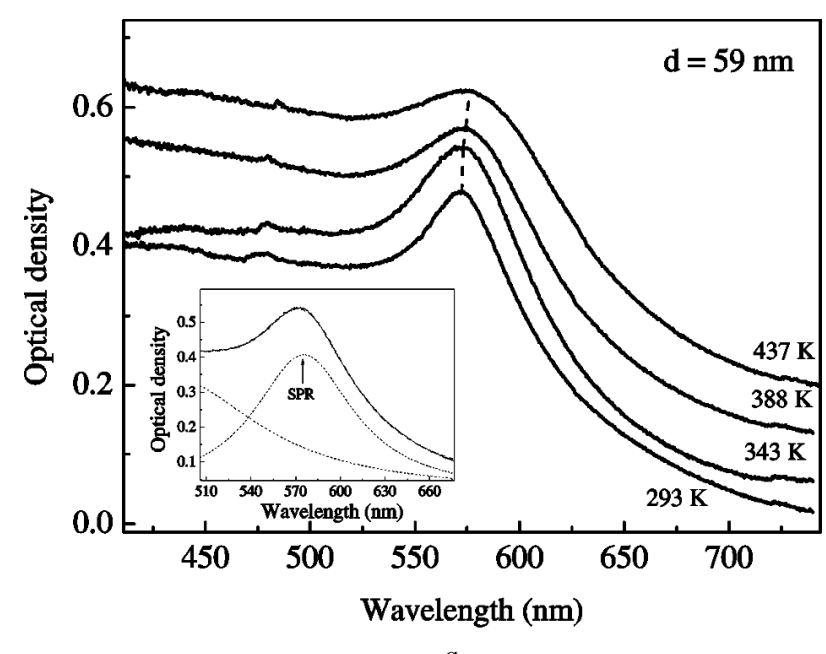

$a$

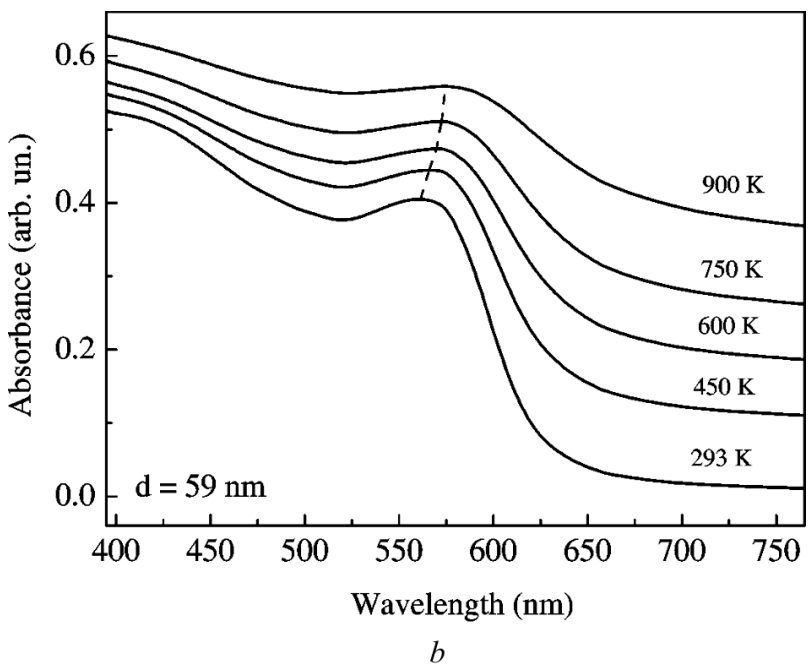

Fig. 2. Measured evolution of the absorption spectrum of $\mathrm{Cu}$ nanoparticles with a mean size of $59 \mathrm{~nm}$ in silica with gradual increase of the temperature from 293 to $437 \mathrm{~K}$ (a). Inset illustrates the decomposition of the total spectrum into basic spectral Lorentzian contours, where SPR is the SP resonance band in a $\mathrm{Cu}$ nanoparticle. Calculated evolution of the absorption spectrum of a $59-\mathrm{nm} \mathrm{Cu}$ nanoparticle in silica with increasing temperature $(b)$

48, and $59 \mathrm{~nm}$ were studied. Absorption spectra were measured in the temperature interval 293-460 K. The aim was to study the effects of the influence of the temperature on the spectral characteristics (energy and bandwidth) of the surface plasmon resonance in copper nanoparticles. Evolution of the experimental absorption spectrum of the composite sample con- taining $\mathrm{Cu}$ nanoparticles with a mean size of $59 \mathrm{~nm}$ is shown in Fig. 2, a.

We performed the decomposition of the total absorption spectra into basic Lorentzian spectral contours. The studied absorption spectra contain two bands. The high-energy band is caused by the interband transitions in copper, and the low-energy one is caused by the excitation of surface plasmons in $\mathrm{Cu}$ nanoparticles. For the studied samples, the SP band is located in the spectral range $570 \mathrm{~nm}(2.18 \mathrm{eV})-580$ $\mathrm{nm}(2.14 \mathrm{eV})$ depending on the nanoparticle size and the temperature. Such spectral position of the SP band is typical of copper nanoparticles embedded in silica [1]. Thus, the spectral decomposition allowed us to extract the surface plasmon band from the total spectrum and determine the spectral characteristics of this band such as the spectral position (energy of SP resonance) and the bandwidth (SP resonance width). The errors of the multipeak analysis were small, specifically the error of determination of the SP energy was about $0.5-0.7 \%$ and one of determination of the SP bandwidth was within $5 \%$. Therefore, the SP band was extracted from the total absorption spectrum quite accurately. This allowed us to obtain width reliable experimental temperature dependences of the SP resonance energy and bandwidth.

Figure 3 presents the obtained temperature dependences of the SP resonance energy and bandwidth for copper nanoparticles of various sizes. Figures 2 and 3 show that the monotonous increase of the temperature from 293 to $460 \mathrm{~K}$ leads to the monotonous red shift (shift to lower frequencies) of the surface plasmon band and to its broadening. It is seen that the manifestations of the temperature effects on the surface plasmon resonance in copper nanoparticles are quite prominent. One can see that the obtained dependences are not qualitatively different for copper nanoparticles with different sizes in the studied size range. Note that the observed temperature dependences for $\mathrm{Cu}$ nanoparticles in silica are quite similar to ones observed for $\mathrm{Ag}$ nanoparticles in the same host matrix [36], i.e. the red shift and the broadening of the SP resonance with increasing temperature. We note that we measured the absorption spectra of $\mathrm{Cu} / \mathrm{SiO}_{2}$ composite samples both at their heating and cooling. We observed the full reversibility of the temperature behavior of the spectra. This indicates the high thermal stability and the high optical quality of sol-gel prepared $\mathrm{Cu} / \mathrm{SiO}_{2}$ nanocomposites. This is 
important for applications of such nanocomposites in optical devices working under extreme thermal conditions.

\section{Temperature Dependence of Surface Plasmon Resonance in Metal Nanoparticles: Theory}

In this section, we give the theoretical analysis of various mechanisms that can cause the observed temperature effects, namely the red shift and the broadening of the SP resonance in metal (copper, in particular) nanoparticles occurring with increasing temperature. Those effects are: (1) electron-phonon scattering in a nanoparticle, (2) thermal expansion of a nanoparticle and (3) temperature dependence of the dielectric permittivity of the silica host matrix.

It is well known (see, e.g., $[1,2]$ ) that the absorption coefficient of a composite with non-interacting spherical metal nanoparticles much smaller than the light wavelength $(d \ll \lambda)$ is

$K(\omega)=\frac{9 f \omega \varepsilon_{m}^{3 / 2}}{c} \frac{\varepsilon_{2}}{\left(\varepsilon_{1}+2 \varepsilon_{m}\right)^{2}+\varepsilon_{2}^{2}}$,

where $\omega$ is the frequency, $c$ is the light velocity, $\varepsilon(\omega)=\varepsilon_{1}(\omega)+i \varepsilon_{2}(\omega)$ is the dielectric permittivity of a nanoparticle, $f$ is the filling factor of the composite, $\varepsilon_{m}$ is the dielectric permittivity of the host matrix. It is clear that the temperature dependence of the permittivities of a nanoparticle and the host matrix would affect the energy and the width of the SP resonance in a nanoparticle and, respectively, affect the absorption spectrum of the composite. The dielectric permittivity of a metal can be expressed as

$\varepsilon(\omega)=\varepsilon_{i b}(\omega)+\varepsilon_{D}(\omega)$,

where $\varepsilon_{i b}(\omega)=\varepsilon_{i b 1}(\omega)+i \varepsilon_{i b 2}(\omega)$ is the contribution of the interband transitions (bound electrons) to the dielectric permittivity of a metal, and $\varepsilon_{\mathrm{D}}(\omega)$ is the contribution of the free electrons that is given by the Drude theory as

$\varepsilon_{\mathrm{D}}(\omega)=1-\frac{\omega_{p}^{2}}{\omega^{2}+i \gamma \omega}$.

Here,

$\omega_{p}=\sqrt{\frac{4 \pi n e^{2}}{m^{*}}}$

ISSN 2071-0194. Ukr. J. Phys. 2013. Vol. 58, No. 3
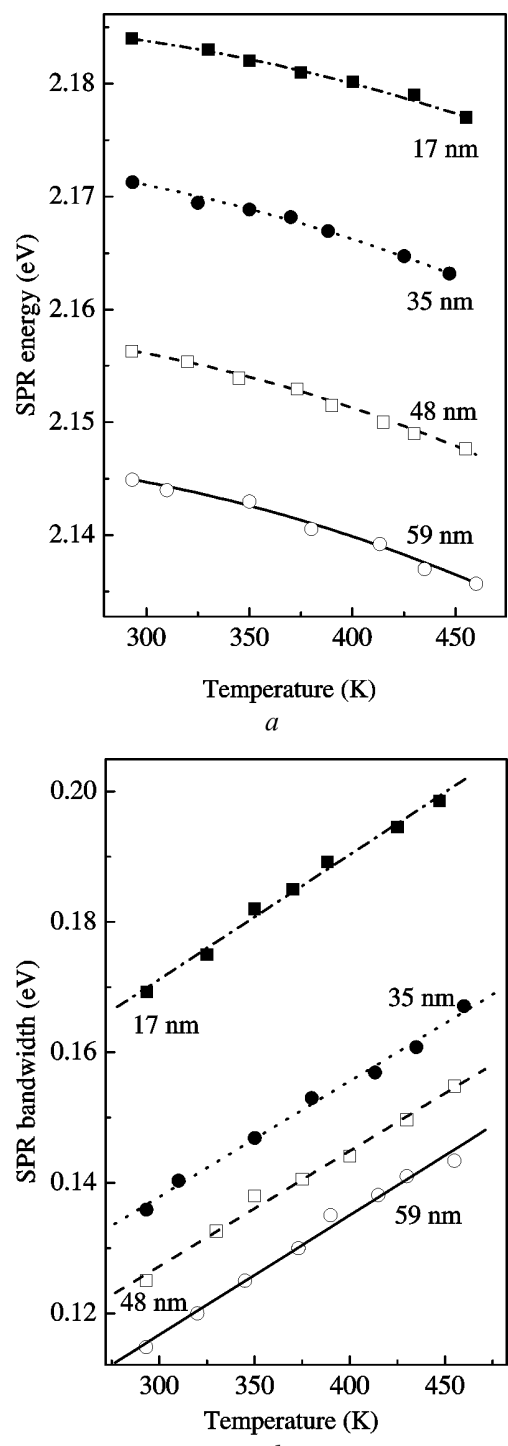

$b$

Fig. 3. Dependences of the SP resonance energy $(a)$ and the bandwidth $(b)$ for copper nanoparticles of various sizes in silica. Points - experiment, lines - theory

is the bulk plasmon frequency, where $n$ is the concentration of free electrons, $e$ is the electron charge, $m^{*}$ is the effective mass of a free electron; $\gamma$ is the damping constant of plasma oscillations. In the approximation of small damping $\left(\varepsilon_{2} \ll\left|\varepsilon_{1}+2 \varepsilon_{m}\right|\right)$, which is quite good for noble metals, the condition of excitation of the SP resonance in the nanoparticles much smaller than the light wavelength is as follows [1]:

$\varepsilon_{1}(\omega)=-2 \varepsilon_{m}$. 
The SP resonance energy is then obtained by substituting the complex dielectric permittivity for a metal nanoparticle from Eqs. (2) and (3) into Eq. (5):

$\omega_{s p}=\sqrt{\frac{\omega_{p}^{2}}{1+\varepsilon_{i b 1}+2 \varepsilon_{m}}-\gamma^{2}}$.

Here, $\varepsilon_{i b 1}$ is the real part of the contribution of interband transitions to the permittivity of a nanoparticle. The plasmon damping constant can be expressed $[1,2]$ as

$\gamma=\gamma_{\infty}+A \frac{v_{\mathrm{F}}}{R}$

where $R$ is the nanoparticle radius, $\gamma_{\infty}$ is the sizeindependent damping constant caused by the scattering of free electrons on electrons, phonons, and lattice defects, $A$ is a theory-dependent parameter that includes details of the scattering process (e.g., isotropic or diffuse scattering $[1,37,38])$, and $v_{\mathrm{F}}$ is the Fermi velocity in a bulk metal $\left(1.57 \times 10^{8} \mathrm{~cm} / \mathrm{s}\right.$ in bulk copper [39]). Note, that there exists a more correct theory of the size dependence of the surface plasmon damping constant $\gamma(R)$ [40]. This theory predicts the oscillating character of $\gamma(R)$, which is a result of the quasidiscrete spectrum of phonons in a nanoparticle. Really, the electron scattering on long-wave phonons dominates in metal, and the long-wave spectrum for metal nanoparticles is limited by the size of a particle that has to lead to oscillations in the $\gamma(R)$ dependence. Note, however, that noticeable oscillations in the dependence $\gamma(R)$ appear for the size of nanoparticles smaller than about $15 \mathrm{~nm}$. For larger particles, the oscillations are small and decrease with increasing size. As a result, the dependence $\gamma(R)$ for larger particles is nearly monotonically decreasing and, respectively, is well described by expression (7). The nanoparticles studied in our work are quite large $(17-59 \mathrm{~nm})$, so our choice of the simplified expression (7) to describe the size dependence of the surface plasmon damping constant is quite correct. Moreover, since the nanoparticles in the samples under study are not monosize, their size distribution has to lead to the blurring of slight oscillations of the damping constant for large nanoparticles.

Let us analyze the influence of the temperature on the energy and the width of the SP resonance in a metal nanoparticle embedded in the host matrix. The first mechanism of the dependence of the SP resonance on the temperature is the electron-phonon scattering in a metal nanoparticle. The size-independent damping constant $\gamma_{\infty}$ depends on the temperature due to the temperature dependence of the electronphonon scattering rate. Really, at an increase of the temperature, the phonon population in a metal increases, which leads to an increase of the probability of the electron-phonon scattering, which results in the increased scattering rate for electrons. The $\gamma_{\infty}(T)$ dependence caused by the electron-phonon scattering is given by [41]

$\gamma_{\infty}(T)=K^{\prime} T^{5} \int_{0}^{\theta / T} \frac{z^{4} d z}{e^{z}-1}$

where $\theta=343 \mathrm{~K}$ is the Debye temperature for copper [39], and $K^{\prime}$ is a constant [41]. Knowing the bulk damping constant $\gamma_{\infty}$ at a certain temperature (e.g., at room one, $\left.T_{0}=293 \mathrm{~K}\right), K^{\prime}$ can be calculated as

$K^{\prime}=\gamma_{\infty}\left(T_{0}\right) /\left(T_{0}^{5} \int_{0}^{\theta / T_{0}} \frac{z^{4} d z}{e^{z}-1}\right)$.

For bulk copper at $T_{0}=293 \mathrm{~K}$, we have $\gamma_{\infty}=0.09$ $\mathrm{eV}$ [42]. Thus, an increase of the electron-phonon scattering rate with the temperature would lead to an increase of the damping constant $\gamma_{\infty}$. Apparently, this would lead to the SP resonance broadening and to its red shift in accordance with Eq. (6).

The second mechanism of the dependence $\varepsilon(T)$ is the thermal expansion of a nanoparticle. Indeed, the nanoparticle volume increases with the temperature,

$V(T)=V_{0}(1+\beta \Delta T)$,

where $\Delta T=T-T_{0}$ is the change in the temperature from room one $\left(T_{0}=293 \mathrm{~K}\right), \beta$ is the volume thermal expansion coefficient $\left(5.1 \times 10^{-5} \mathrm{~K}^{-1}\right.$ for bulk copper [39]), $V_{0}$ is the volume of a nanoparticle at $T_{0}=293 \mathrm{~K}$. The density of free electrons in a metal particle is given by $n=N / V$, where $N$ is the number of electrons, and $V$ is the particle volume. We denote the free electron density at room temperature by $n_{0}$. Since the total number of free electrons in a nanoparticle is temperature-independent [43], $N=n_{0} V_{0}=n(T) V(T)$, we combine Eqs. (4) 
and (10) and obtain the expression for the frequency of a bulk plasmon:

$\omega_{p}=\sqrt{\frac{4 \pi n_{0} e^{2}}{m^{*}(1+\beta \Delta T)}}$.

Substituting Eq. (10) in Eq. (6), we obtain the expression for the frequency of the SP resonance in a metal nanoparticle

$\omega_{s p}=\sqrt{\frac{\omega_{p 0}^{2}}{\left(1+\varepsilon_{i b 1}+2 \varepsilon_{m}\right)(1+\beta \Delta T)}-\gamma^{2}}$,

where $\omega_{p 0}=\sqrt{4 \pi n_{0} e^{2} / m^{*}}$ is the bulk plasmon frequency at room temperature. Thus, the thermal expansion of a nanoparticle would lead to a decrease of the concentration of free electrons in a nanoparticle and, respectively, to a decrease of the SP resonance energy, i.e. to its red shift with increasing temperature. It is well known [1] that the damping constant of plasma oscillations depends on the size of a nanoparticle as $\gamma(R) \propto 1 / R$ (see Eq. (7)). That is due to the scattering of free electrons on the surface of a nanoparticle. Under the thermal expansion, the radius of a nanoparticle increases as

$R(T)=R_{0}(1+\beta \Delta T)^{1 / 3}$,

where $R_{0}$ is the nanoparticle radius at room temperature. Therefore, the thermal expansion of nanoparticles would affect the SP resonance frequency not only through the frequency of a bulk plasmon, but through the size-dependent part of the plasmon damping constant as well. The volume expansion coefficient depends on the temperature according to $[44]$ as

$\beta(T)=\frac{192 \rho k_{\mathrm{B}}}{r_{0} \phi\left(16 \rho-7 T k_{\mathrm{B}}\right)^{2}}$,

where $k_{\mathrm{B}}$ is the Boltzmann constant, and $\rho, \phi$, and $r_{0}$ are parameters of the Morse potential used in Ref. [44] to describe the interatomic interaction potential in metal, $U(r)=\rho\left[e^{-2 \phi\left(r-r_{0}\right)}-2 e^{-\phi\left(r-r_{0}\right)}\right]$.

Note that we consider the thermal expansion of a nanoparticle, by assuming that it is free. However, the nanoparticle is embedded in the silica matrix. Respectively, since the volume thermal expansion coefficient for silica is considerably smaller $\left(1.65 \times 10^{-6} \mathrm{~K}^{-1}\right.$ for fused silica) than one for copper $\left(5.1 \times 10^{-5} \mathrm{~K}^{-1}\right)$, it seems at first glance that the silica host matrix would block the expansion of a nanoparticle. However, our procedure of fabrication of $\mathrm{Cu} / \mathrm{SiO}_{2}$ composite samples is such that the formation of $\mathrm{Cu}$ nanoparticles in silica occurs at the temperature $1473 \mathrm{~K}$, which is considerably higher than the maximum temperature used in our optical measurements $(460 \mathrm{~K})$. So, the sizes of a nanoparticle and a hosting cavity of the silica matrix are equal only at the highest temperature, i.e., at $1473 \mathrm{~K}$. After annealing at $1473 \mathrm{~K}$, the samples were cooled down to room temperature. At cooling, both the nanoparticle and the hosting cavity contracted. But, due to a considerable difference of the coefficients of thermal expansion, the copper nanoparticle contracted considerably stronger, than the hosting cavity did. Thus, at any temperature lower than $1473 \mathrm{~K}$, including the entire temperature range 293-460 K used in our experiments, the copper nanoparticle size is smaller than one of a hosting cavity of the silica matrix. Therefore, we can conclude that the copper nanoparticles in our experiments expanded freely, i.e. the matrix did not affect the thermal expansion of nanoparticles.

At last, the third mechanism of the temperature dependence of the SP resonance can be the temperature dependence of the dielectric permittivity of the host matrix $\varepsilon_{m}(T)$. Indeed, the reference data [45] show that the permittivity of silica increases with the temperature. It is seen from Eq. (12) that such temperature-induced increase of $\varepsilon_{m}$ would lead to a red shift of the SP resonance as well. Thus, summarizing the above arguments, we obtain expressions given below that explain the temperature dependences of the energy and the width of the SP resonance in metal (copper, in particular) nanoparticles in the silica host matrix:

$\omega_{s p}=\sqrt{\frac{\omega_{p 0}^{2}}{\left(1+\varepsilon_{i b 1}+2 \varepsilon_{m}(T)\right)(1+\beta(T) \Delta T)}-\gamma^{2}(T)}$,

$\gamma(T)=\gamma_{\infty}(T)+A \frac{v_{\mathrm{F}}}{R(T)}$.

Here, the dependences $\gamma_{\infty}(T), R(T)$, and $\beta(T)$ are given by Eqs. (8), (13), and (14), respectively. 


\section{Comparison of Experiment and Theory for $\mathrm{Cu}$ Nanoparticles. Discussion}

In this section, we use the theory outlined in the previous section to rationalize the experimental temperature dependences of the SP resonance energy and
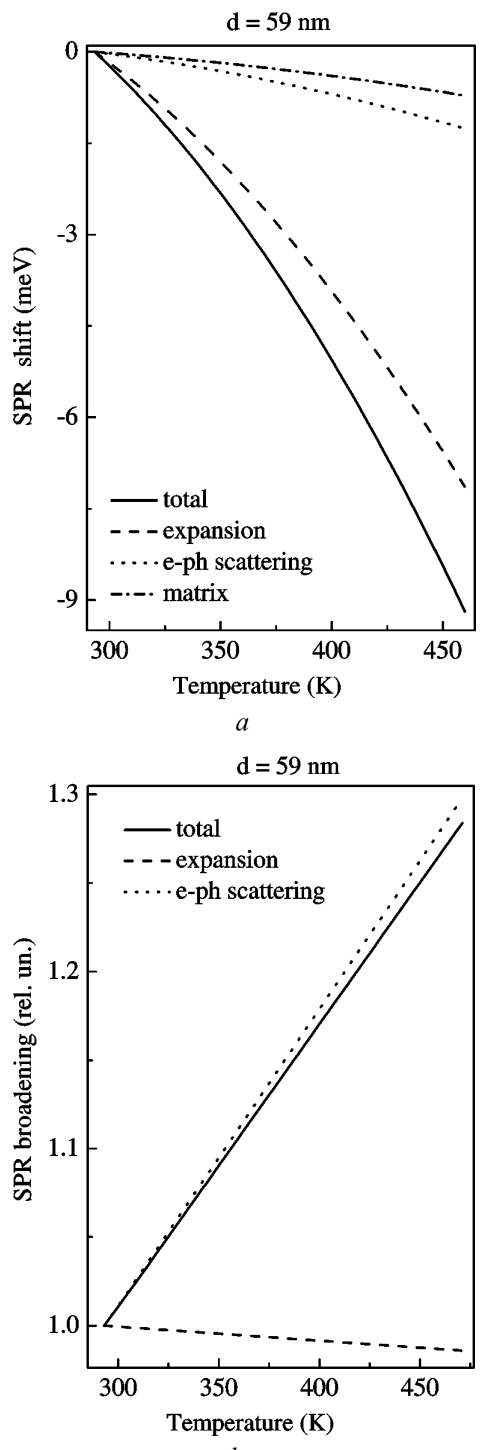

Fig. 4. Calculation of the contributions of different mechanisms to the temperature-induced ( $a$ ) shift and $(b)$ broadening (the ratio $\gamma(T) / \gamma_{293}$ ) of the SP resonance in a 59 -nm copper nanoparticle in silica. Solid lines mark the total shift or broadening, dashed lines - contribution of the thermal expansion of a nanoparticle, dotted lines - the electron-phonon scattering in the nanoparticle, dash-dotted lines - the temperature dependence of the dielectric permittivity of the host matrix bandwidth for copper nanoparticles in silica. We calculate the absorption spectra of $\mathrm{Cu}$ nanoparticles in silica at various temperatures by Eq. (1). The temperature dependence of the silica host matrix was taken from Ref. [45]. To take the temperature dependence of the dielectric permittivity of a nanoparticle into account, we firstly calculate the contribution of interband transitions to $\varepsilon$ of a nanoparticle as

$\varepsilon_{i b}(\omega)=\varepsilon_{\infty}(\omega)-\varepsilon_{\mathrm{D}, \infty}(\omega)$,

where $\varepsilon_{\infty}(\omega)$ is the dielectric permittivity of copper taken from Ref. [46], and $\varepsilon_{\mathrm{D}, \infty}(\omega)$ is the Drude term for bulk copper (contribution of free electrons) calculated by Eq. (3). In calculations, we used $\omega_{p}=7.6 \mathrm{eV}$ and $\gamma_{\infty}=0.09 \mathrm{eV}$ [42]. Then we calculated the temperature-dependent dielectric permittivity of a nanoparticle as follows:

$\varepsilon(\omega, T)=\varepsilon_{i b}(\omega)+\varepsilon_{\mathrm{D}}(\omega, T)$.

Here, $\varepsilon_{\mathrm{D}}(\omega, T)$ was calculated by Eq. (3). In calculations of $\varepsilon_{\mathrm{D}}(\omega, T)$, (1) the temperature-dependent bulk plasmon frequency was calculated as $\omega_{p}=$ $=\omega_{p 0} / \sqrt{1+\beta(T) \Delta T}$, where $\omega_{p 0}=7.6 \mathrm{eV}$, and the volume thermal expansion coefficient was calculated by Eq. (14); (2) the temperature-dependent damping constant $\gamma(T)$ was calculated by Eq. (16), where $\gamma_{\infty}(T)$ was calculated by Eqs. (8) and (9), and $R(T)$ was determined from Eq. (13). In calculations, we also used $v_{\mathrm{F}}=1.57 \times 10^{8} \mathrm{~cm} / \mathrm{s}$ for the Fermi velocity in bulk copper, $A=0.11$ was estimated from the fitting of the experimental size dependence of the $\mathrm{SP}$ resonance bandwidth at $T_{0}=293 \mathrm{~K}$, and the parameters of the Morse potential $\rho=0.3287 \mathrm{eV}$, $\phi=13.123 \mathrm{~nm}^{-1}$, and $r_{0}=0.28985 \mathrm{~nm}$ were taken from Ref. [47].

The evolution of the calculated absorption spectrum of 59-nm copper nanoparticles in silica with increasing temperature is presented in Fig. $2 b$. It is seen that an increase of the temperature has to lead to the red shift and the broadening of the surface plasmon band. This trend is similar to one observed experimentally. To check quantitatively our above assumptions of the physical mechanisms of these two phenomena, we also calculated the temperature dependences of the shift and the broadening of the SP resonance in copper nanoparticles. To compare the respective experimental and calculated dependences properly, we obtained the theoretical val- 
ues of SP energy and bandwidth by the decomposition of the calculated spectra to the basic spectral Lorentzian contours, since the corresponding experimental values we determined from the decomposition of the experimental spectra. The obtained calculated temperature dependences of the SP resonance energy and bandwidth are presented in Fig. 3. The respective experimental dependences are shown in this figure as well. It is seen that a quite good agreement of the experimental and calculated dependences takes place. This fact proves the correctness of our theoretical model.

As was noted above, the theoretically calculated dependences contain contributions of three mechanisms. Those are the electron-phonon scattering in a nanoparticle, thermal expansion of a nanoparticle, and temperature dependence of the dielectric permittivity of the host matrix. It is important to know the relative contribution of each mechanism to the total temperature effect. To do so, we performed such calculations for the temperature dependences of the SP resonance energy and width. The results for 59-nm $\mathrm{Cu}$ nanoparticles in silica are presented in Fig. 4. It is seen from Fig. 4, a that the thermal expansion is the dominant mechanism of the temperature-induced red shift of the SP resonance in copper nanoparticles. Contributions of the increase of both the electronphonon scattering rate and the dielectric permittivity of the host matrix with increasing temperature are close to each other and are quite small as compared with the contribution of the thermal expansion. Meanwhile, the dominant mechanism of the temperature-induced broadening of the SP resonance in copper nanoparticles is the electron-phonon scattering, which is seen from Fig. 4, $b$. Thermal expansion of a nanoparticle leads to a very small decrease of the SP resonance width. However, this decrease is negligibly small and can be neglected. The temperature dependence of the dielectric permittivity of the host matrix does not affect the SP resonance width. Note that the good agreement of experiments with the above-outlined theory takes place as well for the temperature dependence of the SP resonance in silver nanoparticles [36]. This proves the similarity of the physical mechanisms of the observed temperature dependences for SP resonances in copper and silver nanoparticles, i.e. the thermal expansion of a nanoparticle as a cause of the red shift and the electron-phonon scattering as a cause of the broadening of the $\mathrm{SP}$ resonance. It is seen from Fig. 3, a that the red shift rate of the SP resonance with increasing temperature is the same for $\mathrm{Cu}$ nanoparticles of all studied sizes. This indicates that the volume thermal expansion coefficient is size-independent in the studied range of $\mathrm{Cu}$ nanoparticle sizes.

\section{Conclusions}

The temperature dependences of the SP resonance energy and width in copper nanoparticles with mean sizes of $17,35,48$, and $59 \mathrm{~nm}$ embedded in the silica glass host matrix are studied in the temperature range $293-460 \mathrm{~K}$. We observed that, as the temperature increases, the red shift and the broadening of the SP resonance occur. A theoretical model including the phenomena of electron-phonon scattering in a nanoparticle, the thermal expansion of the nanoparticles, and the temperature dependence of the dielectric permittivity of the host matrix is considered to rationalize the observed temperature behavior of the SP resonance in copper nanoparticles. As the temperature of a particle increases, the nanoparticle volume increases, and the density of free electrons decreases. The lower electron density leads to the lower plasma frequency of electrons and subsequently to the red shift of the SP resonance. The rate of electron-phonon scattering increases with the temperature. This leads to an increase of the damping constant of plasma oscillations and, as a result, to the red shift of the SP resonance. The dielectric permittivity of silica increases with the temperature, which leads to a red shift of the SP resonance as well. The results of calculations of the temperature dependences of the SP resonance energy and width are in very good agreement with the respective experimental data, which proves the validity of the used theoretical model. It is shown that the thermal expansion of copper nanoparticles is the dominant mechanism of the temperature-induced red shift of the SP resonance in copper nanoparticles. Meanwhile, the dominant mechanism of the SP resonance broadening with increasing temperature is the electron-phonon scattering in $\mathrm{Cu}$ nanoparticles.

The author thanks Dr. A.A. Alexeenko for samples of $\mathrm{Cu} / \mathrm{SiO}_{2}$ nanocomposite, Dr. A.M. Dmytruk for TEM measurements, and Prof. I.M. Dmitruk for fruitful discussions. 
1. U. Kreibig and M. Vollmer, Optical Properties of Metal Clusters (Springer, Berlin, 1995).

2. C.F. Bohren and D.R. Huffman, Absorption and Scattering of Light by Small Particles (Wiley, Chichester, 1998).

3. B.G. Ershov, E. Janata, A. Henglein, and A. Fojtik, J Phys. Chem. 97, 4589 (1993).

4. A. Henglein, J. Phys. Chem. 97, 5457 (1993).

5. A. Barhoumi, D. Zhang, F. Tam, and N. Halas, J. Am. Chem. Soc. 130, 5523 (2008).

6. F. Le, D. Brandl, Y. Urzhumov, H. Wang, J. Kundu, N. Halas, J. Aizpurua, and P. Nordlander, ACS Nano 2, 707 (2008).

7. G. Laurent, N. Felidj, J. Grand, J. Aubard, G. Levi, A. Hohenau, J. Krenn, and F. Aussenegg, J. of Microsc.-Oxford 229, 189 (2008).

8. R. Bakker, H. Yuan, Z. Liu, V. Drachev, A. Kildishev, V. Shalaev, R. Pedersen, S. Gresillon, and A. Boltasseva, Appl. Phys. Lett. 92, 043101 (2008).

9. G. Gay, B. de Lesegno, R. Mathevet, J. Weiner, H. Lezec, and T. Ebbesen, Appl. Phys. B 81, 871 (2005).

10. O.A. Yeshchenko, I.M. Dmitruk, A.A. Alexeenko, M.Yu. Losytskyy, A.V. Kotko, and A.O. Pinchuk, Phys. Rev. B 79, 235438 (2009).

11. A. Gobin, M. Lee, R. Drezek, N. Halas, and J. West, Clin. Cancer Res. 11, 9095S (2005).

12. C. Hubert, A. Rumyantseva, G. Lerondel, J. Grand, S. Kostcheev, L. Billot, A. Vial, R. Bachelot, and P. Royer, Nano Lett. 5, 615 (2005).

13. K. Kandere-Grzybowska, C. Campbell, Y. Komarova, B. Grzybowski, and G. Borisy, Nature Methods 2, 739 (2005).

14. M. Choi, K.J. Stanton-Maxey, J.K. Stanley, C.S. Levin, R. Bardhan, D. Akin, S. Badve, J. Sturgis, J.P. Robinson, R. Bashir, N.J. Halas, and S.E. Clare, Nano Lett. 7, 3759 (2007).

15. L. Hirsch, A. Gobin, A. Lowery, F. Tam, R. Drezek, N. Halas, and J. West, Annals Biomed. Engineering 34, 15 (2006).

16. D. O'Neal, L. Hirsch, N. Halas, J. Payne, and J. West, Cancer Lett. 209, 171 (2004).

17. D. Citrin, Nano Lett. 5, 985 (2005).

18. J. Jung, T. Sondergaard, and S. Bozhevolnyi, Phys. Rev. B 76, 035434 (2007).

19. K. Leosson, T. Nikolajsen, A. Boltasseva, and S. Bozhevolnyi, Opt. Express 14, 314 (2006).

20. B. Steinberger, A. Hohenau, H. Ditlbacher, A. Stepanov, A. Drezet, F. Aussenegg, A. Leitner, and J. Krenn, Appl. Phys. Lett. 88, 094104 (2006).
21. J. Takahara, S. Yamagishi, H. Taki, A. Morimoto, and T. Kobayashi, Opt. Lett. 22, 475 (1997).

22. U. Kreibig, Appl. Phys. B 93, 79 (2008).

23. W.A. Challener, C. Peng, A.V. Itagi, D. Karns, W. Peng, Y. Peng, X.M. Yang, X. Zhu, N.J. Gokemeijer, Y.-T. Hsia, G. Ju, R.E. Rottmayer, M.A. Seigler, and E.C. Gage, Nature Photon. 3, 303 (2009).

24. L.R. Hirsch, R.J. Stafford, J.A. Bankson, S.R. Sershen, B. Rivera, R.E. Price, J.D. Hazle, N.J. Halas, and J.L. West, Proc. Natl. Acad. Sci. USA 100, 13549 (2003).

25. A. Lowery, A. Gobin, E. Day, N. Halas, and J. West, Breast Cancer Res. Treat. 100, S289 (2006).

26. A. Lowery, A. Gobin, E. Day, N. Halas, and J. West, Int. J. Nanomed. 1, 149 (2006).

27. L. Cao, D.N. Barsic, A.R. Guichard, and M.L. Brongersma, Nano Lett. 7, 3523 (2007).

28. W. Cai, J.S. White, and M.L. Brongersma, Nano Lett. 9, 4403 (2009).

29. U. Kreibig, J. Phys. F 4, 999 (1974).

30. R.H. Doremus, J. Chem. Phys. 40, 2389 (1964).

31. R.H. Doremus, J. Chem. Phys. 42, 414 (1965).

32. P. Mulvaney, in Nanoscale Materials in Chemistry, edited by K.J. Klabunde (Wiley, New York, 2001), p. 121.

33. J.-S.G. Bouillard, W. Dickson, D.P. O'Connor, G.A. Wurtz, and A.V. Zayats, Nano Lett. 12, 1561 (2012).

34. D.Yu. Fedyanin, A.V. Krasavin, A.V. Arsenin, and A.V. Zayats, Nano Lett. 12, 2459 (2012).

35. S. Link and M.A. El-Sayed, J. Phys. Chem. B 103, 4212 (1999)

36. O.A. Yeshchenko, I. M. Dmitruk, A.A. Alexeenko, A.V. Kotko, J. Verdal, and A.O. Pinchuk, Plasmonics 7, 685 (2012).

37. U. Kreibig and U. Genzel, Surf. Sci. 156, 678 (1985).

38. S. Link and M. El-Sayed, J. Phys. Chem. B 103, 8410 (1999).

39. C. Kittel, Introduction to Solid State Physics (Wiley, New York, 2005).

40. N.I. Grigorchuk and P.M. Tomchuk, Phys. Rev. B 84 085448 (2011)

41. K. Ujihara, J Appl. Phys. 43, 2374 (1972).

42. N.W. Ashcroft and N. D. Mermin, Solid State Physics (Saunders College, Philadelphia, 1976).

43. R.H. Bube, Electrons in Solids: An Introductory Survey (Academic Press, London, 1992).

44. Z. Li-Jun, G. Jian-Gang, and Z. Ya-Pu, Chin. Phys. Lett. 26, 066201 (2009).

ISSN 2071-0194. Ukr. J. Phys. 2013. Vol. 58, No. 3 
45. J.H. Wray and J.T. Neu, J. Opt. Soc. Am. 59, 774 (1969).

46. P.B. Johnson and R.W. Christy, Phys. Rev. B 6, 4370 (1972).

47. R.C. Lincoln, K.M. Koliwad, and P.B. Ghate, Phys. Rev. 157, 463 (1967).

Received 22.11.12

О.А. Єщенко

ВПЛИВ ТЕМПЕРАТУРИ

НА ПОВЕРХНЕВИЙ ПЛАЗМОННИЙ

РЕЗОНАНС В НАНОЧАСТИНКАХ МІДІ

Р е $з$ ю м е

Досліджено температурні залежності енергї та півширини поверхневого плазмонного резонансу в сферичних наночастинках міді розмірами 17-59 нм у кварцевій матриці в ін- тервалі температур 293-460 К. Показано, що підвищення температури приводить до червоного зсуву та розширення поверхневого плазмонного резонансу в наночастинках $\mathrm{Cu}$. Отримані залежності проаналізовано в рамках теоретичної моделі, яка включає теплове розширення наночастинки, електрон-фононне розсіяння у наночастинці та температурну залежність діелектричної проникності матриці. Показано, що теплове розширення є основним механізмом, що зумовлює температурний червоний зсув поверхневого плазмонного резонансу в наночастинках міді. Виявлено, що об'ємний коефіцієнт теплового розширення наночастинок $\mathrm{Cu}$ не залежить від розміру частинки в дослідженому діапазоні розмірів. У свою чергу показано, що збільшення частоти електрон-фононного розсіяння при збільшенні температури є домінуючим механізмом розширення поверхневого плазмонного резонансу в наночастинках міді. 